\title{
Applications of minimal surfaces to the topology of three-manifolds
}

\author{
William H. Meeks, III
}

\section{Introduction}

In this paper, I will mention some applications of minimal surfaces to the geometry and topology of three-manifolds that I discussed in my lecture at the Current Developments in Mathematics Conference for 2004.

The first important application of minimal surfaces to the geometry of three-manifolds was given by Schoen and Yau [22] in their study of Riemannian three-manifolds of positive scalar curvature and their related proof of the positive mass conjecture in general relativity. The techniques that they developed in their proof of this conjecture continue to be useful in studying relationships between stable minimal surfaces and the topology of Riemannian manifolds.

Around 1978, Meeks and Yau gave geometric versions of three classical theorems in three-dimensional topology. These classical theorems concern the existence of certain embedded surfaces. In the geometric versions of these theorems, Meeks and Yau proved the existence of essentially cononical solutions, which are given by area minimizing surfaces. They referred to these theorems as the Geometric Dehn's Lemma, Geometric Loop Theorem and the Geometric Sphere Theorem.

As an application of these special geometric minimal surface solutions to these classical topological theorems, Meeks and Yau gave new equivariant versions of these theorems in the presence of a differential finite group action. Their Equivariant Loop Theorem turned out to be the final missing step in the solution of the Smith Conjecture concerning the standardness of the smooth action of finite cyclic groups on the

This material is based upon work by the NSF under Award No. DMS - 0405836. Any opinions, findings, and conclusions or recommendations expressed in this publication are those of the authors and do not necessarily reflect the views of the NSF. 
three-sphere $S^{3}$. These and related results will be discussed in Section 2 .

Recently, Colding and Minicozzi [1] gave an application of minimal surfaces to study the Ricci flow on a closed Riemannian three-manifold that is a homotopy sphere. They proved that on such a manifold the Ricci flow has finite extinction time, which means that a one-parameter family $g(t)$ of metrics evolving by Ricci flow becomes singular in finite time. A sketch of their proof of this result appears in Section 3.

\section{Embedded least-area surfaces in three-manifolds}

In this section, I will review some of the results and background material for the minimal surface analogues of the classical Dehn's Lemma, Loop Theorem and the Sphere Theorem in three-manifold topology. We begin this discussion with the statement of the minimal surface version of Dehn's Lemma by Meeks and Yau. We recall that the least-energy map referred to in the statement of the theorem below has least-area and is conformal on the interior of $D$, where $D$ is the unit disk in $\mathbb{C}$.

Theorem 2.1 (Geometric Dehn's Lemma [13]). Let $M$ be a compact three-manifold with convex boundary. If $\Gamma$ is a simple closed curve in $\partial M$ which is homotopically trivial in $M$, then:

(1) There exists a map $f: D \rightarrow M$ of least-energy such that $\left.f\right|_{\partial D}$ is a parametrization of $\Gamma$.

(2) Any map $f: D \rightarrow M$ given in (1) is injective and a smooth immersion of the interior of $D$.

(3) Such an $f$ is as regular as $\Gamma$ along $\partial D$ and if $\Gamma$ is of class $C^{2}$, then $f$ is an immersion.

(4) If $f_{1}$ and $f_{2}$ are two such solutions and $f_{1}(\operatorname{Int}(D)) \bigcap f_{2}(\operatorname{Int}(D))$ $\neq \varnothing$, then $f_{2}=f_{1} \circ \varphi$, where $\varphi$ is a conformal diffeomorphism of $D$.

The proof of the existence of a least-energy $f: D \rightarrow M$ follows from Morrey's solution of Plateau's problem in a homogeneously regular $n$ manifold (without boundary). We now sketch how this result follows. In the case $\partial M$ is smooth and strictly convex, we proved that $M$ embeds as a subdomain of a homogeneously regular three-manifold $\widetilde{M}$ such that any compact minimal surface $\Sigma$ in $\widetilde{M}$, with $\partial \Sigma \subset M \subset \widetilde{M}$, is contained in $M$. Hence, the Morrey least-energy solution to Plateau's problem for a $\Gamma \subset M \subset \widetilde{M}$ in $\widetilde{M}$, actually is contained in $M$. For the general case where $\partial M$ is geodesically convex and perhaps just continuous, then one uses an approximation procedure to obtain a Morrey solution to the classical Plateau problem for $\Gamma \subset \partial M$, which has finite least-energy.

By Osserman [19] and Gulliver [4], one obtains that $\left.f\right|_{\operatorname{Int}(D)}$ is an immersion. Statement 3 that the immersion $f$ is as regular as $\partial D$ follows from results of Lewy $[\mathbf{9}]$ in the case $\Gamma$ is analytic, and when $\Gamma$ is of 
class $C^{2}$ from results of Hildebrandt [7]. The nonexistence of boundary branch points for $f: D \rightarrow M$, when $\Gamma$ is of class $C^{2}$, easily follows from the $C^{2}$-regularity of $f$ and the convexity of $\partial M$.

The proof of statement 2 in the case $\Gamma$ and $M$ are analytic is given by a topological argument, called the tower construction, used to prove the classical Dehn's Lemma in three-manifold topology. We give the proof of this analytic case at the end of this section. The proof of injectivity in the case of a general $\Gamma$ and a general $M$ with convex boundary is accomplished by approximation arguments.

The proof of statement 4 in the analytic case is a straightforward modification of an argument similar to the one used in the proof of statement 2 in the analytic case. This argument is based on a cut and paste argument, which we now explain. Suppose that $D_{1}, D_{2}$ are two least-area embedded disks in $M$ with $\partial D_{1}=\partial D_{2}=\Gamma$, which intersect transversely at some interior point of the disks. Then, there is a simple closed curve $\gamma$ in the intersection which bounds subdisks $\widetilde{D}_{1} \subset D_{1}$ and $\widetilde{D}_{2} \subset D_{2}$. Without loss of generality, we may assume that Area $\left(\widetilde{D}_{1}\right) \leq$ Area $\left(\widetilde{D}_{2}\right)$. Then cut $\widetilde{D}_{2}$ out of $D_{2}$ and replace it by $\widetilde{D}_{1}$, to obtain a piecewise smooth disk $\widetilde{D}_{2}=\left(D_{2}-\widetilde{D}_{2}\right) \cup \widetilde{D}_{1}$ with $\widehat{D}_{2}=\Gamma$ which is not smooth, but has at most the same area as $D_{2}$. But, the area of $\widehat{D}_{2}$ can be decreased along $\gamma$, and so, a small perturbation of $\widehat{D}_{2}$ has less area than $D_{2}$, contradicting our least-area assumption for $D_{2}$.

Since every compact three-manifold has a smooth metric that is a product metric in a small $\varepsilon$-product neighborhood of its boundary, every compact three-manifold admits a metric with convex boundary. Thus, statement 2 yields the classical topological result.

Corollary 2.2 (Dehn's Lemma). A smooth simple closed curve on the boundary of a three-manifold, which is homotopically trivial in the three-manifold, is the boundary of a smooth embedded disk.

One of our original motivations for proving our Geometric Dehn's Lemma was to prove the following now classical result.

COROllary $2.3([\mathbf{1 3}])$. Let $\Gamma$ be a simple closed curve in $\mathbb{R}^{3}$ that is extremal (it lies on the boundary of its convex hull). Then, $\Gamma$ bounds a disk of finite area and any classical Douglas solution to Plateau's problem for $\Gamma$ is an embedded minimal disk.

We now discuss the free boundary value problem that arises in our proof of the Geometric Loop Theorem. For this, we first consider a special easier to visualize case. Consider a three-manifold $M$ which is a smooth solid torus (possibly knotted) in $\mathbb{R}^{3}$ whose boundary has nonnegative mean curvature. Courant [3] considered the classical free boundary valued problem for $M$ and proved that there exists a branched minimal disk $f: D \rightarrow M$ of least-area such that $f(\partial D)$ represents a homotopically nontrivial curve in the boundary torus $\partial M$. The following 
theorem shows that such an $f$ is a smooth embedding with $f(D)$ orthogonal to $\partial M$ along $\partial D$.

TheOrem 2.4 ([14] and [12]). Let $M$ be a compact three-manifold, whose boundary has nonnegative mean curvature. Let $S$ be the disjoint union of some components of $\partial M$. Let $K$ be the kernel of the map $i_{*}: \pi_{1}(S) \rightarrow \pi_{1}(M)$, where $i$ is the inclusion map. Then:

(1) There are a finite number of smooth conformal maps $f_{1}, \ldots, f_{k}$ from the unit disk $D$ into $M$, so that,

(a) $f_{1}$ has least-area among all maps from $D$ into $M$ whose boundary $\sigma_{1}$ represents a nontrivial element in $K$.

(b) For each $i, f_{i}$ has minimal area among all maps from $D$ into $M$ whose boundary $\sigma_{1}$ does not belong to the smallest normal subgroup of $\pi_{1}(S)$ containing $\left[\sigma_{1}\right], \ldots,\left[\sigma_{i-1}\right]$.

(c) The disks $f_{i}(D)$ are orthogonal to $\partial M$ along their boundary $\sigma_{i}$.

(d) $K$ is the smallest normal subgroup of $\pi_{1}(S)$ containing $\left[\sigma_{1}\right], \ldots,\left[\sigma_{k}\right]$.

(2) Any set of conformal mappings $f_{1}, \ldots, f_{k}$ satisfying properties (a) and (b) are embeddings and have mutually disjoint images.

(3) If $g_{1}, \ldots, g_{l}$ is another set of conformal mappings satisfying (a) and (b), then any two mappings in the set $\left\{f_{1}, \ldots, f_{k}, g_{1}, \ldots, g_{l}\right\}$ either are equal up to conformal reparametrization or have disjoint images.

We just remark that the strategy in the proof of the Geometric Loop Theorem is similar to the proof of the Geometric Dehn's Lemma in most respects. However, in the proof of the Geometric Loop Theorem, we needed to prove the existence of a least-energy solution $f: D \rightarrow M$ to the free boundary value problem; in the previous case, we could refer more directly to Morrey's solution to the classical Plateau problem.

The above theorem has the following topological corollary.

Corollary 2.5 (Loop Theorem). If $M$ is a three-manifold and there exists a homotopically nontrivial curve in $\partial M$, which is homotopically trivial in $M$. Then, there exists an embedded disk $(D, \partial D) \subset$ $(M, \partial M)$ with $\partial D$ homotopically nontrivial in $\partial M$.

Since minimal surfaces are rather cononical, our geometric methods have potential applications beyond those obtained by the classical topological solutions. Indeed, a moment's thought shows that applications of geometric solutions to study smooth compact group actions will be most fruitful because these groups can be considered as groups of isometries of some Riemannian metric and minimal surfaces must behave well under such actions. In this way, we were able to prove Dehn's Lemma, Loop Theorem and the Sphere Theorem (to be discussed) in equivariant form. Combining an observation of Gordan and Literland, 
the following equivariant loop theorem and a theorem of W. Thurston (which also depends on a theorem of H. Bass), one settled in the affirmative the conjecture of P.A. Smith on the unknottedness of the fixed point set of a finite cyclic group action on $S^{3}$ (see [15] for details).

Corollary 2.6 (Equivariant Loop Theorem ([12])). If $G$ is a smooth finite group action on a compact three-manifold $M$ with compressible boundary (some homotopy nontrivial curve in $\partial M$ is homotopically trivial in $M)$, then there exists an embedded disk $(D, \partial D) \subset$ $(M, \partial M)$ with $\partial D$ homotopically nontrivial in $\partial M$ such that the $G$ orbit of $D$ is an embedded two-manifold.

We now state the Geometric Sphere Theorem and its corresponding Equivariant Sphere Theorem as a corollary.

TheOREM 2.7 (Geometric Sphere Theorem [12]). Let $M$ be a threemanifold with convex boundary. Then, there exist conformal maps $f_{1}$, $\ldots, f_{k}$ from $S^{2}$ into $M$ such that:

(1) $f_{1}: S^{2} \rightarrow M$ is homotopically nontrivial and minimizes area among all homotopically nontrivial maps from $S^{2}$ into $M$. For each $i, f_{i}$ does not belong to the $\pi_{1}(M)$ submodule of $\pi_{2}(M)$ generated by $\left\{f_{1}, \ldots, f_{i-1}\right\}$ and $f_{i}$ minimizes area among all such maps.

(2) $\left\{f_{1}, \ldots, f_{k}\right\}$ generates $\pi_{2}(M)$ as a $\pi_{1}(M)$ module.

(3) For any set of maps $\left\{g_{1}, \ldots, g_{l}\right\}$ from $S^{2}$ into $M$ that satisfy property (1), then $g_{i}$ is either a conformal embedding or a twoto-one covering map whose image is an embedded real projective plane $R P^{2}$. Furthermore, if $\left\{f_{1}, \ldots, f_{k}\right\}$ and $\left\{g_{1}, \ldots, g_{l}\right\}$ are two sets of mappings satisfying property (1), then, for all $i$ and $j$, either the images of $f_{i}$ and $g_{j}$ are disjoint or $f_{i}$ and $g_{j}$ are equal up to conformal reparametrization.

THEOREM 2.8. Suppose $M$ is a compact orientable three-manifold and

$$
M=\left(\begin{array}{c}
k_{1} \\
\# P_{1} \\
i=1
\end{array}\right) \#\left(\begin{array}{c}
k_{2} \\
\# P_{2} \\
i=1
\end{array}\right) \# \ldots \#\left(\begin{array}{c}
k_{n} \\
\# P_{n} \\
i=1
\end{array}\right) \text {, where \# denotes }
$$
connected sum and $P_{1}, P_{2}, \ldots, P_{n}$ are distinct prime orientable threemanifolds such that $P_{i}$ is not homotopically equivalent to $S^{3}$ or $S^{2} \times S^{1}$. Suppose there is a finite group $G$ of diffeomorphisms acting effectively on $M$. Then:

(1) There is a natural homomorphism $\sigma: G \rightarrow \Pi_{i=1}^{n} S\left(k_{i}\right)$, where $S\left(k_{i}\right)$ is the permutation group on $k_{i}$ letters.

(2) There is a natural injective homomorphism $\tau: \operatorname{Ker}(\sigma) \rightarrow$ $\Pi_{i=1}^{n} \operatorname{Diff}\left(P_{i}\right)$. 
(3) Let $\pi_{j}: \prod_{i=1}^{n} S\left(k_{i}\right) \rightarrow S\left(k_{j}\right)$ be the projection on the $j^{\text {th }}$ coordinate. Then, there is a natural injective homomorphism $\alpha: \operatorname{Ker}\left(\pi_{j} \circ \sigma\right) \rightarrow \operatorname{Diff}\left(P_{j}\right)$.

(4) If $k_{1}=k_{2}=\ldots k_{n}=1$, then $G$ act effectively on $S^{2}$ and effectively on each $P_{j}$ as a finite group of diffeomorphisms with some fixed point. In particular, in this case $G$ is isomorphic to a finite subgroup of the orthogonal group $O(3)$.

Our equivariant sphere theorem also shows that for a finite group acting smoothly on the connected sum of compact nonsimply connected prime orientable manifolds with fundamental group nonisomorphic to the integers, the action must split equivariantly up to the permutations of the factors. Hence, basically when we study finite group actions on a three-manifold, we can assume the manifold is prime. The sphere theorem also enables us to deal with finite groups acting on noncompact manifolds. For example, combining the above mentioned affirmative answer to the Smith conjecture and the sphere theorem, we prove in [15] that finite cyclic groups acting smoothly on $\mathbb{R}^{3}$ must be conjugate to the linear action and that every finite subgroup of $\operatorname{Diff}\left(\mathbb{R}^{3}\right)$ is isomorphic to a subgroup of $O(3)$. In fact, these results and further work by Thurston show that every finite subgroup of $\operatorname{Diff}\left(\mathbb{R}^{3}\right)$ is conjugate to a subgroup of $O(3) \subset \operatorname{Diff}\left(\mathbb{R}^{3}\right)$. In $[\mathbf{1 1}]$, we generalized Theorem 2.8 to the case where $P_{i}$ is not $S^{3}$ but may be a homotopy three-sphere.

2.1. The proof of the embedding of the analytic case of the Geometric Dehn's Lemma. In this section, we give a simplified proof of the basic topological construction used in the proof of the geometric Dehn's Lemma in [13]. We give the proof only in the analytic setting.

TheOREM 2.9. Suppose $M$ is a compact analytic Riemannian threemanifold. Suppose that $D$ is the closed unit disk in the plane and $\gamma$ is an analytic curve on $\partial M$ and that $f: D \rightarrow M$ is a least-area (energy) map with $f(\partial D)=f(D) \cap \partial N=\gamma$. Then $f$ is injective.

The proof of the theorem will depend on the following sequence of lemmas.

Lemma 2.10. $f: D \rightarrow M$ is an analytic immersion.

Proof. By the regularity theorems of Gulliver [4] and Osserman [19], $f$ is an immersion on the interior of the $D$. The function $f$ is analytic on $\operatorname{Int}(D)$ by Morrey's interior regularity theorem [18]. The map $f$ is analytic on $D$ by the boundary regularity theorems by Lewy [9] and by Hildebrandt [7]. By a theorem of Gulliver-Lesley [5], $f$ is an immersion on $D$.

Lemma 2.11. $f: D \rightarrow M$ is simplicial with respect to fixed triangulations of $D$ and $M$. 
Proof. By Lemma 2.10, $f$ is analytic and it follows that $f(D)$ is a semi-analytic subset of $M$. Also, it follows from the triangulation theorems in [10] that the semi-analytic subset $f(D)$ of $M$ is a twodimensional subcomplex of some triangulation of $M$. Since $f$ is an immersion, the triangulation of $f(D)$ induces a triangulation of $D$ such that $f: D \rightarrow M$ is simplicial.

LEMma 2.12. Suppose $D_{1}$ and $D_{2}$ are distinct analytic embedded disks in an open Riemannian three-manifold $N$ and that $D_{1}$ and $D_{2}$ have least-area with respect to their boundary curves. If $D_{1} \cap D_{2} \subset$ $\operatorname{Int}\left(D_{1}\right) \cap \operatorname{Int}\left(D_{2}\right)$, then $D_{1} \cap D_{2}=\varnothing$.

Proof. Suppose first that $D_{1}$ and $D_{2}$ are in general position, which is the generic case. If $D_{1} \cap D_{2}$ is nonempty, then $D_{1} \cap D_{2}$ is a compact one-dimensional submanifold of $\operatorname{Int}\left(D_{1}\right)$ and of $\operatorname{Int}\left(D_{2}\right)$. By the classification of one-dimensional submanifolds, $D_{1} \cap D_{2}$ is a finite collection of simple closed curves. Let $\gamma$ be a component in $D_{1} \cap D_{2}$. Then the Jordan curve theorem implies that $\gamma$ is the boundary of a subdisk $\widetilde{D}_{1}$ of $D_{1}$ and a subdisk $\widetilde{D}_{2}$ of $D_{2}$.

Without loss of generality, suppose that the area of $\widetilde{D}_{1}$ is less than or equal to the area of $\widetilde{D}_{2}$. Then consider the new piecewise smooth disk:

$$
D_{3}=\left(D_{2}-\widetilde{D_{2}}\right) \cup \widetilde{D_{1}} .
$$

The area of $D_{3}$ is less than or equal to the area $D_{2}$. The area of $D_{3}$ can now be decreased along $\gamma$, which contradicts the hypothesis that $D_{2}$ has least-area with respect to its boundary curve.

If $D_{1}$ and $D_{2}$ are not in general position, then there are two ways to reduce to the general position case. The first way is by approximation. The second is by way of the following assertion:

Assertion 2.13. If $D_{1} \cap D_{2} \subset \operatorname{Int}\left(D_{1}\right) \cap \operatorname{Int}\left(D_{2}\right)$ is nonempty, then $D_{1} \cap D_{2}$ contains a simple closed curve.

Proof of Assertion 2.13. Since $D_{1}$ and $D_{2}$ are analytic, $\Gamma=$ $D_{1} \cap D_{2}$ is a compact triangulable analytic subset of $\operatorname{Int}\left(D_{1}\right)$. We first note that $\Gamma$ has no isolated vertices. If $\Gamma$ had an isolated vertex $p$, then $p$ would correspond to a point on $D_{1}$ where $D_{1}$ is locally on one side of $D_{2}$. By the maximum principal for minimal surfaces, $D_{1}$ and $D_{2}$ intersect in an open set near $p$, and so, the vertex $p$ is not isolated. Also, $\Gamma$ cannot contain a 2 -simplex, because by the uniqueness of analytic continuation, $D_{1}$ and $D_{2}$ must agree on an open set that goes to the boundary of $D_{1}$ or $D_{2}$. However, this is impossible, since the intersection of $D_{1}$ and $D_{2}$ does not, by hypothesis, include points on the boundaries.

The argument used above shows that $\Gamma$ is a one-dimensional subcomplex of some triangulation of $D_{1}$ and $\Gamma$ contains no isolated vertices. Analytic one-dimensional subsets of a disk have an even number of edges 
at every vertex. This implies that $\Gamma$ represents a one-cycle in the simplicial one-chains of $D_{1}$ using $\mathbb{Z}_{2}$-coefficients. Since the first homology group with $\mathbb{Z}_{2}$ coefficients of $D_{1}$ is zero, geometric intersection theory implies that $\Gamma$ must disconnect $D_{1}$. A boundary curve of an inner-most component of $D_{1}-\Gamma$ is the required simple closed curve in the assertion, and so, the assertion is proved.

We now return to the proof of Lemma 2.12. The existence of a simple closed curve in $D_{1} \cap D_{2}$ together with the disk replacement argument used in the general position case gives a contradiction. Hence, $D_{1} \cap D_{2}$ must be empty, which proves the lemma.

Lemma 2.14. Suppose $N$ is a triangulated three-dimensional manifold and $f: D \rightarrow N$ is a simplicial immersion of a disk with respect to some triangulation $T$ of $D$. Then there exists a subdivision of the triangulation of $N$, so that $f: D \rightarrow N$ is still simplicial with respect to $T$ and such that the simplicial neighborhood of $f(D)$ is a simplicial regular neighborhood of $f(D)$. The simplicial neighborhood of $f(D)$ is the union of the simplices which intersect $f(D)$.

Proof. This elementary result follows after subdividing two times the triangulation of $N$. Each time the subdivision includes the baricenters of the simplices which are not contained in $f(D)$. This proves Lemma 2.14.

We now carry out the construction of a tower for $f: D \rightarrow M$ in order to simplify the self-intersection or singular set for $f: D \rightarrow M$, which by Lemma 2.11 is simplicial. First, let $N_{1}$ be a simplicial regular neighborhood of $f(D)$ given in Lemma 2.14. After restricting the range space of $f$ to $N_{1}$, there is a new map $f_{1}: D \rightarrow N_{1}$. If $N_{1}$ is not simply connected, then let $P_{1}: \widetilde{N}_{1} \rightarrow N_{1}$ be the universal covering space of $N_{1}$ and let $\widetilde{f}_{1}: D \rightarrow \widetilde{N}_{1}$ be a lift of $f_{1}$ to this covering space. Then restricting the range space of $\widetilde{f}_{1}$ to a regular neighborhood $N_{2}$ of $\widetilde{f}_{1}(D)$, we get another map $f_{2}: D \rightarrow N_{2}$.

If $N_{2}$ is not simply connected, then we can repeat the construction in the previous paragraph to get a lift $\widetilde{f}_{2}: D \rightarrow \widetilde{N}_{2}$ to the universal covering space $P_{2}: \widetilde{N}_{2} \rightarrow N_{2}$ of $N_{2}$. After restricting the lift $\widetilde{f}_{2}$ to the regular neighborhood $N_{3}$ of $\widetilde{f}(D)$, we get $f_{3}: D \rightarrow N_{3}$.

Repeating $k$-times, the construction outlined above yields a tower

$$
P_{k} \circ P_{k-1} \circ \ldots \circ P_{1}: N_{k} \rightarrow N_{1},
$$

where $P_{i}: N_{i+1} \rightarrow N_{i}$ is the restriction of $P_{i}: \widetilde{N}_{i} \rightarrow N_{i}$ to $N_{i+1}$.

Each $N_{i}$ in the above tower is a Riemannian manifold with respect to the pulled back metric. Each of the lifts $f_{i}: D \rightarrow N_{i}$ is a solution to Plateau's problem for the simple closed curve $f_{i}(\partial D)$ with respect to this metric. Otherwise, there is an immersion $g: D \rightarrow N_{i}$ with $g(\partial D)=$ 
$f_{i}(\partial D)$ and with respect to the pulled back metric on $D$, Area $(g)<$ Area $\left(f_{i}\right)=$ Area $(f)$, which is impossible.

By Lemmas 2.11 and 2.14, we may assume that each map $f_{i}: D \rightarrow$ $N_{i}$ in the tower is simplicial with respect to a fixed triangulation $T$ for which $f_{i}: D \rightarrow N_{1}$ is simplicial. Note that the triangulation on $N_{i}$ is induced from the triangulation on $N_{i-1}$ pulled back to $\widetilde{N}_{i}$ by $P_{i}: \widetilde{N}_{i} \rightarrow N_{i}$. We now use this fact to prove that the tower construction terminates, after some finite number $n$ of steps, with $N_{k}$ being simply connected, where $n$ is at most equal to the number of simplices in $T \times T$. We will consider $T$ to be a collection of open simplices and vertices.

LEMmA 2.15. If $S\left(f_{i}\right)=\{(\sigma, \tau) \in T \times T \mid \sigma \neq \tau$ and $f(\sigma)=f(\tau)\}$, then $S\left(f_{i+1}\right)$ is a proper subset of $S\left(f_{i}\right)$. Hence, the tower construction terminates at some $k$ with $N_{k}$ simply connected.

Proof. Since $f_{i}=P_{i} \circ f_{i+1}$ where $P_{i}$ is a simplicial map, then $S\left(f_{i+1}\right) \subset S\left(f_{i}\right)$. If $S\left(f_{i+1}\right)=S\left(f_{i}\right)$, then $h=\left.P_{i}\right|_{f_{i+1}(D)}$ induces a homeomorphism between $f_{i+1}(D)$ and $f_{i}(D)$. Using $h$ we can define a lift of the inclusion map $i: f_{i}(D) \rightarrow N_{i}$ to $\widetilde{N}_{i}$ by $\widetilde{i}: f_{i}(D) \rightarrow \widetilde{N}_{i}$, where $\widetilde{i}=h^{-1} \circ i$. Since $N_{i}$ is a regular neighborhood of $f_{i}(D)$, then $i_{*}: \pi_{1}\left(f_{i}(D)\right) \rightarrow \pi_{1}\left(N_{i}\right)$ is an isomorphism. Since $\tilde{N}_{i}$ is simply connected, the lifting criterion for maps in covering space theory implies that $N_{i}$ is simply connected. Thus, we may assume that $S\left(f_{i+1}\right)<$ $S\left(f_{i}\right)$, which proves the lemma.

Lemma 2.16. The lift $f_{k}: D \rightarrow N_{k}$ is one-to-one.

We first show:

Assertion 2.17. The boundary of $N_{k}$ consists of spheres.

Proof. Since $N_{k}$ is simply connected, $H_{1}\left(N_{k}, \mathbb{Z}_{2}\right)=0$. Since the pairing between homology and cohomology with coefficients in a field is non-degenerate, $H^{1}\left(N_{k}, \mathbb{Z}_{2}\right)=0$. Poincare duality then shows that $H_{2}\left(N_{k}, \partial N_{k}, \mathbb{Z}_{2}\right)=0$. From the following part of the long exact sequence in homology for the pair $\left(N_{k}, \partial N_{k}\right)$,

$$
\rightarrow H_{2}\left(N_{k}, \partial N_{k}, \mathbb{Z}_{2}\right) \rightarrow H_{1}\left(\partial N_{k}, \mathbb{Z}_{2}\right) \rightarrow H_{1}\left(N_{k}, \mathbb{Z}_{2}\right) \rightarrow
$$

one computes that $H_{1}\left(\partial N_{k}, \mathbb{Z}_{2}\right)=0$. This shows that the first homology group with $\mathbb{Z}_{2}$ coefficients is zero for each boundary component of $N_{k}$. By the classification theorem for compact surfaces, each component of the boundary of $N_{k}$ is a sphere, which proves the assertion.

Proof. We now prove Lemma 2.16. We shall now use the fact that the boundary of $N_{k}$ consists entirely of spheres to show that $f_{k}: D \rightarrow$ $N_{k}$ is an embedding. First note that since $N_{k}$ is a simplicial regular neighborhood, there is, after a subdivision, a simplicial retraction $S: N_{k} \rightarrow f_{k}(D)$ whose restriction $R=\left.S\right|_{\partial N_{k}} \rightarrow f_{k}(D)$ has the following 
property: $R$ covers each open two simplex of $f_{k}(D)$ exactly two times and $R$ restricted to $\partial N_{k}-f_{k}(\partial D)$ is locally one-to-one. The existence of such a retraction follows directly from the definition of a simplicial regular neighborhood and the collapsing properties of such a neighborhood onto an immersed codimension-one simplicial submanifold whose boundary is the intersection of the submanifold with the boundary of the ambient manifold. For a proof of the existence, we refer the reader to $[\mathbf{1 6}]$.

By Assertion 2.17, the curve $\gamma_{k}=f_{k}(\partial D)$ is contained in a sphere $S$ in $\partial N_{k}$. The Jordan curve theorem implies that the simple closed curve $\gamma_{k}$ disconnects the sphere $S$ into two disks $D_{1}$ and $D_{2}$. Now consider the following inequalities:

$$
\text { Area }\left(\left.R\right|_{D_{1}}\right)+\operatorname{Area}\left(\left.R\right|_{D_{2}}\right) \leq \operatorname{Area}\left(\left.R\right|_{\partial N_{k}}\right) \leq 2 \text { Area }\left(f_{k}\right) .
$$

The last inequality follows from the fact that area is carried by twosimplices and $\left.F\right|_{\partial N_{k}}$ covers each two-simplex of $f_{k}(D)$ twice.

Since $f_{k}$ is a solution to Plateau's problem for $\gamma_{k}$, the above area inequality implies that $\left.R\right|_{D_{1}}$ and $\left.R\right|_{D_{2}}$ are also disks of least-area with $\gamma_{k}$ for boundary. However, if $f_{k}$ is not an embedding, then the area of $\left.R\right|_{D_{1}}$ and $\left.R\right|_{D_{2}}$ can be decreased along a self-intersection curve of $f_{k}(D)$. Since this contradicts the least-area property of $f_{k}$, the map $f_{k}$ must be an embedding, which proves the lemma.

We now complete the proof of Theorem 2.9. If $f: D \rightarrow M$ is not an embedding, then we may assume by the previous lemma that $k$ is greater than one and $f_{k-1}: D \rightarrow N_{k-1}$ is not one-to-one. Let $E$ be the embedded disk $i \circ f_{k}(D) \subset \widetilde{N}_{k-1}$, where $i: N_{k} \rightarrow \widetilde{N}_{k-1}$ is the inclusion map. Since $f_{k-1}$ is not one-to-one and $N_{k-1}=\widetilde{N}_{k-1} / G$ where $G$ is the group of covering transformations, then there exists a nontrivial covering transformation $\tau: \widetilde{N}_{k-1} \rightarrow \widetilde{N}_{k-1}$ such that $\tau(E) \cap E$ is nonempty. Since the covering transformation $\tau$ is an isometry of $\widetilde{N}_{k-1}$, the disk $\tau(E)$ has least-area with respect to its boundary curve. The hypothesis in the theorem that $f(\partial D)=f(D) \cap \partial M=\gamma$ implies that $E \cap \tau(E) \subset$ $\operatorname{Int}(E) \cap \tau(\operatorname{Int}(E))$. Lemma 2.12 shows this containment is impossible, which implies that $f: D \rightarrow M$ must in fact be an embedding. This completes the proof of Theorem 2.9 .

\section{Application of minimal surfaces to the problem of finite extinction time for the Ricci flow}

In this section, we review some results on minimal surfaces by Colding and Minicozzi that have an application to the question of finite extinction time for the Ricci flow on certain Riemannian three-manifolds. These results and related discussion are taken from the papers in $[\mathbf{1}]$ and $[\mathbf{2}]$. 
Let $M$ be a smooth closed orientable three-manifold and let $g(t)$ be a one-parameter family of metrics on $M$ evolving by the Ricci flow, so

$$
\partial_{t} g=-2 \operatorname{Ric}_{M_{t}}
$$

For the remainder of this section, we will assume that $M$ is a prime three-manifold, which is nonaspherical, which just means that some homotopy group $\pi_{k}(M)$ is nonzero for some $k>1$. Recall that a closed orientable three-manifold is irreducible if every embedded two-sphere in the manifold is the boundary of a ball. Note that $S^{2} \times S^{1}$ is the only compact orientable three-manifold which is prime but not irreducible. If $M$ is irreducible, then the sphere theorem in the previous section implies $\pi_{2}(M)=0$, and the Hurewicz isomorphism theorem implies in this case that $\pi_{3}(M) \neq 0$. Since $\pi_{3}\left(S^{2} \times S^{1}\right)=\pi_{3}\left(S^{2}\right)=\mathbb{Z}$, we see that for the manifold in the case we are considering, $\pi_{3}(M) \neq 0$.

Consider the space of continuous maps from $S^{2}$ to $M$. This space is naturally a fiber bundle over $M$. Using this fact and suspension on the long exact sequence of related homotopy groups, Micallif and Moore, in Lemma 3 in $[\mathbf{1 7}]$, proved that this space is not simply connected.

Fix a continuous map

$$
\beta:[0,1] \rightarrow C^{0} \cap L_{1}^{2}\left(S^{2}, M\right),
$$

where $\beta(0)$ and $\beta(1)$ are constant maps and so that $\beta$ is in the nontrivial homotopy class $[\beta]$. We define the width $W=W(g,[\beta])$ by

$$
W(g)=\min _{\gamma \in[\beta]} \max _{s \in[0,1]} \operatorname{Energy}(\gamma(s)) .
$$

The next theorem, Theorem 0.3 in [1], gives an upper bound for the derivative of $W(g(t))$ under the Ricci flow, which forces the solution $g(t)$ to become extinct in finite time. We remark that Perelmann [20] has also found a proof that $g(t)$ becomes extinct in finite time in this situation.

ThEOREM 3.1 ([1] and [20]). Let $M$ be a closed orientable prime nonaspherical three-manifold equipped with a Riemannian metric $g=$ $g(0)$. Under the Ricci flow, the width $W(g(t))$ satisfies

$$
\frac{d}{d t} W(g(t)) \leq-4 \pi+\frac{3}{4(t+C)} W(g(t)),
$$

in the sense of the limsup of forward difference quotients. Hence, $g(t)$ must become extinct in finite time.

Suppose that $\Sigma \subset M$ is a closed immersed surface (not necessarily minimal); then results of Hamilton [6] give

$$
\left.\frac{d}{d t}\right|_{t=0} \operatorname{Area}_{g(t)}(\Sigma)=-\int_{\Sigma}\left[R-\operatorname{Ric}_{M}(\mathbf{n}, \mathbf{n})\right] .
$$


If $\Sigma$ is also minimal, then

$$
\begin{aligned}
\left.\frac{d}{d t}\right|_{t=0} \operatorname{Area}_{g(t)}(\Sigma) & =-2 \int_{\Sigma} K_{\Sigma}-\int_{\Sigma}\left[|A|^{2}+\operatorname{Ric}_{M}(\mathbf{n}, \mathbf{n})\right] \\
& =-\int_{\Sigma} K_{\Sigma}-\frac{1}{2} \int_{\Sigma}\left[|A|^{2}+R\right] .
\end{aligned}
$$

Here, $K_{\Sigma}$ is the Gaussian curvature of $\Sigma$, and $\mathbf{n}$ is a unit normal for $\Sigma$. $A$ is the second fundamental form of $\Sigma$, so that $|A|^{2}$ is the sum of the squares of the principal curvatures, $\operatorname{Ric}_{M}$ is the Ricci curvature of $M$, and $R$ is the scalar curvature of $M$. (The curvature is normalized so that on the unit $S^{3}$ the Ricci curvature is 2 and the scalar curvature is 6.) To get the above equation, one uses that by the Gauss equations and minimality of $\Sigma$

$$
\mathrm{K}_{\Sigma}=\mathrm{K}_{M}-\frac{1}{2}|A|^{2},
$$

where $\mathrm{K}_{M}$ is the sectional curvature of $M$ on the two-plane tangent to $\Sigma$.

The first lemma in $[\mathbf{1}]$ gives an upper bound for the rate of change of area of minimal two-spheres, and we give their proof of it.

Lemma 3.2. If $\Sigma \subset M$ is a branched minimal immersion of the two-sphere, then

$$
\left.\frac{d}{d t}\right|_{t=0} \operatorname{Area}_{g(t)}(\Sigma) \leq-4 \pi-\frac{\operatorname{Area}_{g(0)}(\Sigma)}{2} \min _{M} R(0) .
$$

Proof. Let $\left\{p_{i}\right\}$ be the set of branch points of $\Sigma$ and $b_{i}>0$ the order of branching at $p_{i}$. From above, we have

$$
\left.\frac{d}{d t}\right|_{t=0} \operatorname{Area}_{g(t)}(\Sigma) \leq-\int_{\Sigma} K_{\Sigma}-\frac{1}{2} \int_{\Sigma} R=-4 \pi-2 \pi \sum b_{i}-\frac{1}{2} \int_{\Sigma} R,
$$

where the equality used the Gauss-Bonnet theorem with branch points.

The evolution equation for the scalar curvature $R=R(t)$ of $M_{t}$ under Ricci flow (see $[\mathbf{6}]$ ) is given by the following equation and gives rise to a related inequality:

$$
\partial_{t} R=\Delta R+2|\operatorname{Ric}|^{2} \geq \Delta R+\frac{2}{3} R^{2}
$$

A maximum principle argument then gives for some constant $C$,

$$
R(t) \geq-\frac{3}{2(t+C)} .
$$

Plugging this estimate for $R(t)$ into Lemma 3.2 then yields:

$$
\left.\frac{d}{d t}\right|_{t=0} \operatorname{Area}_{g(0)}(\Sigma) \leq-4 \pi+\frac{3 \operatorname{Area}(\Sigma)}{4(t+C)} .
$$

What Colding and Minicozzi do next is derive a related forward difference quotient for $W(g(t))$. Namely, they show that there is a constant 
$\widetilde{C}$, so that, given $\varepsilon>0$, there exists an $\bar{h}>0$ such that for $0<h<\bar{h}$, then

$$
\frac{W(g(\tau+h))-W(g(\tau))}{h} \leq-4 \pi+\widetilde{C} \varepsilon+\frac{3}{4(\tau+C)} W(g(\tau))+\widetilde{C} h .
$$

Taking $\varepsilon \rightarrow 0$ gives the differential inequality in Theorem 3.1.

Colding and Minicozzi derive the above related forward difference quotient inequality by applying the previous estimate for the derivative of the areas of minimal two-spheres $\Sigma$ which arise in the next proposition. This proposition asserts the existence of a special sequence $\gamma^{i}$ of sweep-outs, where for some $s_{j}, s_{j} \in[0,1]$, the spheres $\gamma_{s_{j}}^{j}$ converge to a collection of branched minimal spheres with total energy $W(g)$. This proposition is Theorem 4.2.1 by Jost in [8]. This result depends on the theory of minimal spheres using the concept of $\alpha$-energy and the fact that $\alpha$-energy functional is a Morse function on the appropriate spaces. This theory was first developed by Sachs and Uhlenbeck [21] with improvements by Meeks and Yau [12] and Siu and Yau (see Chapter VIII in $[\mathbf{2 3}]$ to prove that there was no loss of energy in the limit as $\alpha \rightarrow 0$ ). The index-one bound for the minimal spheres described below is not stated explicitly in $[\mathbf{8}]$ but follows by the arguments in $[\mathbf{1 7}]$.

Proposition 3.3. Given a metric $g$ on $M$ and a nontrivial $[\beta] \in$ $\pi_{1}\left(C^{0} \cap L_{1}^{2}\left(S^{2}, M\right)\right)$, there exists a sequence of sweep-outs $\gamma^{j}:[0,1] \rightarrow$ $C^{0} \cap L_{1}^{2}\left(S^{2}, M\right)$ with $\gamma^{j} \in[\beta]$ so that

$$
W(g)=\lim _{j \rightarrow \infty} \max _{s \in[0,1]} \operatorname{Energy}\left(\gamma_{s}^{j}\right) .
$$

Furthermore, there exist $s_{j} \in[0,1]$ and branched conformal minimal immersions $u_{0}, \ldots, u_{m}: S^{2} \rightarrow M$ with index at most one so that, as $j \rightarrow \infty$, the maps $\gamma_{s_{j}}^{j}$, converge to $u_{0}$ weakly in $S_{1}^{2}$ and uniformly on compact subsets of $S^{2} /\left\{x_{1}, \ldots, x_{k}\right\}$, and

$$
W(g)=\sum_{i=0}^{m} \operatorname{Energy}\left(u_{i}\right)=\lim _{j \rightarrow \infty} \operatorname{Energy}\left(\gamma_{s_{j}}^{j}\right) .
$$

Finally, for each $e>0$, there exists a point $x_{k_{i}}$ and a sequence of conformal dilations $D_{i, j}: S^{2} \rightarrow S^{2}$ about $x_{k_{i}}$, so that the maps $\gamma_{s_{j}}^{j} \circ D_{i, j}$ converge to $u_{i}$.

Finally, we show that the differential inequality for $W(g(t))$ given in Theorem 3.1 implies finite extinction time for the Ricci flow. Namely, rewriting this inequality as $\frac{d}{d t}\left(W(g(t))(t+C)^{3 / 4}\right) \leq-4 \pi(t+C)^{-3 / 4}$ and then integrating gives

$$
(T+C)^{-3 / 4} W(g(T)) \leq C^{-3 / 4} W(g(0))-16 \pi\left[(T+C)^{1 / 4}-C^{1 / 4}\right] .
$$

Since $W \geq 0$ by definition and the right hand side of the equation would become negative for $T$ sufficiently large, the theorem follows. 


\section{References}

[1] T.H. Colding and W.P. Minicozzi II, Estimates for the extinction time for the Ricci flow on certain 3-manifolds and a question of Perelman, JAMS 18, 347559.

[2] T.H. Colding and W.P. Minicozzi II, An excursion into geometric analysis, in 'Surveys of Differential Geometry IX - Eigenvalues of Laplacian and other geometric operators' (A. Grigor'yan and S.-T. Yau, eds.), International Press, 2004, 83-146.

[3] R. Courant, Dirichlet's Principle, Conformal Mapping and Minimal Surfaces, Interscience Publishers, Inc., New York, 1950.

[4] R. Gulliver, Regularity of minimizing surfaces of prescribed mean curvature, Ann. of Math. 97 (1973), 275-305, MR0317188, Zbl 0246.53053.

[5] R. Gulliver and F. Lesley, On boundary branch points of minimizing surfaces, Arch. Rational Mech. Anal. 52 (1973), 20-25, MR0346641, Zbl 0263.53009.

[6] R.S. Hamilton, The formation of singularities in the Ricci flow, in 'Surveys in differential geometry', Vol. II (Cambridge, MA, 1993), 1-119, International Press, Cambridge, MA, 1995.

[7] S. Hildebrandt, Boundary behavior of minimal surfaces, Archive Rational Mech. Anal. 35 (1969), 47-81.

[8] J. Jost, Two-dimensional geometric variational problems, J. Wiley and Sons, Chichester, NY, 1991.

[9] H. Lewy, On the boundary behavior of minimal surfaces, Proceedings of the National Academy 37 (1951), 103-110.

[10] S. Lojasiewicz, Triangulation of semianalytic sets, Ann. Scuola Norm. Sup. Pisa 18 (1964), 449-474.

[11] W.H. Meeks III, L. Simon, and S.-T. Yau, The existence of embedded minimal surfaces, exotic spheres and positive Ricci curvature, Annals of Math. 116 (1982), 221-259.

[12] W.H. Meeks III and S.-T. Yau, Topology of three dimensional manifolds and the embedding problems in minimal surface theory, Annals of Math. 112 (1980), 441-484.

[13] W.H. Meeks III and S.-T. Yau, The classical Plateau problem and the topology of three-dimensional manifolds, Topology 21(4) (1982), 409-442.

[14] W.H. Meeks III and S.-T. Yau, The existence of embedded minimal surfaces and the problem of uniqueness, Math. Z. 179 (1982), 151-168.

[15] W.H. Meeks III and S.-T. Yau, Compact group actions on $\mathbb{R}^{3}$, in 'Conference on the Smith Conjecture', Academic Press, 1984.

[16] W.H. Meeks III and S.-T. Yau, The topological uniqueness of complete minimal surfaces of finite topological type, Topology 31(2) (1992), 305-316.

[17] M. Micallef and J.D. Moore, Minimal two-spheres and the topology of manifolds with positive curvature on totally isotropic two-planes, Annals of Math. 127 (1988), 199-227.

[18] C.B. Morrey, The problem of Plateau in a Riemannian manifold, Annals of Math. 49 (1948), 807-851.

[19] R. Osserman, A proof of the regularity everywhere to Plateau's problem, Annals of Math. 91(2) (1970), 550-569, MR0266070, Zbl 0194.22302.

[20] G. Perelman, Finite extinction time for the solutions to the Ricci flow on certain three-manifolds, math.DG/0307245.

[21] J. Sacks and K. Uhlenbeck, Minimal immersions of closed Riemannian surfaces, Transactions of the AMS 271 (1982), 639-652. 
[22] R. Schoen and S.-T. Yau, Existence of incompressible minimal surfaces and the topology of three dimensional manifolds with non-negative scalar curvature, Annals of Math. 110 (1979), 127-142.

[23] R. Schoen and S.-T. Yau, Lectures on harmonic maps, International Press, 1997.

Mathematics Department, University of Massachusetts

AMHERST, MA 01003

E-mail address: bill@math.umass.edu 
